\title{
EL TRABAJO COMUNAL EN LA UNIVERSIDAD DE COSTA RICA (CASO: GESTIÓN AMBIENTAL EN PUNTARENAS)
}

\section{THE STUDENT COMMUNITY WORK AT THE UNIVERSITY OF COSTA RICA (CASE: ENVIRONMENTAL ADMINISTRATION IN PUNTARENAS)}

\section{Kathia García Cousin*}

RESUMEN

La Universidad de Costa Rica en la Sede del Pacífico desarrolla el Proyecto de Trabajo Comunal Universitario (TCU) dirigido a la Gestión Ambiental al Nivel Comunal. Este contribuye con la formación de una conciencia crítica sobre el problema del medio ambiente en la provincia de Puntarenas. El presente artículo pretende conocer el grado de pertinencia de este proyecto de TCU durante el período 2003-2007, para optar por posibles opciones de mejoramiento en la calidad de vida de los habitantes de la zona.

PALABRAS CLAVE: UNIVERSIDAD DE COSTA RICA * ACCION SOCIAL * TCU * MEDIO AMBIENTE $*$ COMUNIDADES

\section{ABSTRACTS}

Universidad de Costa Rica, Sede del Pacífico develops a student's Community Work (TCU) named: "Environmental administration at Community Level" which contributes with the formation of an environmental-educative conscience of the residents of Puntarenas city. This article seeks to know the degree of relevancy of this project of TCU during the period 2003-2007, to offer alternatives of improvement of the inhabitants' quality of life in Puntarenas.

KEY WORDS: UNIVERSITY OF COSTA RICA $*$ SOCIAL ACTION $*$ TCU * ENVIRONMENT * COMMUNITIES

Profesora Coordinadora Tcu, Sede del Pacífico,

Universidad de Costa Rica.

kcousin4@yahoo.es 


\section{INTRODUCCIÓN}

Hoy se vive la paulatina desaparición de valores $^{1}$ y se percibe a la sociedad con apatía hacia la conservación de la naturaleza. Pero basta una breve alteración del medio ambiente, para que se rompa el equilibrio del entorno $y$ las personas empiecen a preocuparse. Surge entonces la necesidad de utilizar racionalmente los recursos naturales, para garantizar la supervivencia de las personas en este planeta.

Sierra (2005) considera que la Tierra viene afrontando problemas tales como: la deforestación, la contaminación, el calentamiento global. Es alarmante que en América Latina cerca de un $15 \%$ de la población, o sea unos 76 millones de personas, no tengan acceso a agua potable (se mide por el porcentaje de la población que utiliza fuentes de agua tratada y mejorada). Este preciado líquido empieza a desaparecer $y$ a contaminarse por el uso $y$ abuso, ya que los bosques son maltratados y eliminados del medio. Es preocupante también, que ya en el 2007, Costa Rica presente déficit de madera $y$, no tenga previsto programas dirigidos a la producción de árboles específicamente, a la demanda nacional de este insumo, para la fabricación de muebles.

Las relaciones causales del deterioro ambiental siguen patrones de procesos económicos, industriales, petroleros y químicos exagerados por la idea equivocada de considerarlos recursos inagotables.

En la actualidad, existen ciudades muy contaminadas en el mundo. Un estudio realizado por la Organización Mundial de la Salud (OMS), y el Programa de las Naciones Unidas para el Medio Ambiente (PNUMA), acerca de la contaminación del aire en 20 urbes de las 24 del mundo (áreas urbanas con más de 10 millones de habitantes), precisa que la Ciudad de México es la más contaminada del planeta, y registra niveles graves de dióxido de sulfuro $\left(\mathrm{SO}_{2}\right)$ con

1 Entiéndase los valores en el sentido ético: "Conjunto de normas morales que rigen la conducta humana". RAE. Diccionario de la Lengua Española. 22. ${ }^{\circ}$ edición. 2001 (avance de la 23. ${ }^{\circ}$ edición) www.rae.es partículas en suspensión (SPM), monóxido de carbono (CO) y ozono tropos feérico $\left(\mathrm{O}_{3}\right)$; asî como altos niveles de plomo (РВ) y óxido de nitrógeno $\left(\mathrm{NO}_{2}\right)$. Además puntualiza que 13 de las 15 urbes más contaminadas del planeta están en Asia y, siguiendo con la lista, aparecen otras ciudades con graves niveles de contaminación tales como: Bangkok, Beijing, Bombay, Buenos Aires, Calcuta, Delhi, Karachi, Londres, Manila, Área Metropolitana de Nueva York, Río de Janeiro, Seúl, Shangai y Tokio² .

Ahora bien, a fin de salvar las cuentas se necesitan medidas urgentes para resguardar la vida en la Tierra, y mediante el Protocolo de Kioto $^{3}$ se trata de salvar al mundo preservando la subsistencia en él; sin embargo, resulta contraproducente que países como Estados Unidos, el cual produce un $36,1 \%$ del total de estos gases, no esté en ese Protocolo.

En Costa Rica, Arce (2005) explica que el país dedica el $4 \%$ de su territorio a parques nacionales $y$ áreas protegidas $y$ aporta la venta de oxígeno a naciones como Noruega, amparado a una convención de cambio climático. Por ende, estas actividades costarricenses de acuerdo con Mediavilla (2007) tratan de minimizar el problema del calentamiento global. Según el último informe de Panel Intergubernamental (IPCC) de las Naciones Unidas, la temperatura media del planeta subirá 1,9 y 4,6 grados en el siglo XXI si no corregimos a tiempo, ocasionaría el descongelamiento de las masas polares. En pocas palabras, subirá el nivel del mar y provocará consecuentemente la muerte de corales, así como el aumento de acidez en el agua, y por supuesto las costas y llanuras serán las más vulnerables a este fenómeno.

Afortunadamente para los habitantes de la provincia de Puntarenas, Costa Rica, esta zona se encuentra rodeada de asombrosos ecosistemas naturales como lo son: humedales,

OMS/PNUMA. "Las ciudades más contaminadas del mundo". En: <htpp:/www.ecoestrategia.com/ articulos/mapas/articulos/mapas $02 . h t m l>$ Consultado 5 de marzo del 2007.

3 Tratado Internacional de Ambiente: obliga a los países a reducir sus emisiones de carbono en un 5\% por debajo de los niveles de 1990-2018. 
manglares $y$ esteros con abundantes riquezas marinas. Además, desarrolla una importante actividad agrícola con los diversos cultivos como la caña de azúcar, el melón y la sandía. En las partes altas: Esparza y Miramar, producen chile y tomate; la zona de Peñas Blancas, siembra café y en el campo de la actividad fruticultora produce mango, aguacate, limón mecina, guanábana y naranjas. Cabe señalar que la principal actividad económica de esta provincia es la pesca.

Sin embargo, a partir de la década de los años ochenta, el Cantón Central de la provincia ha venido experimentando un acelerado crecimiento urbano $y$ no ha planificado el tratamiento de desechos sólidos divididos en orgánicos (descomposición rápida) e inorgánicos (descomposición extremadamente lenta, tales como el plástico, el vidrio, los metales, etc.).

Por otra parte, la Municipalidad de Puntarenas advierte que recoge aproximadamente 155 toneladas de basura por día y 40 toneladas más de basura industrial; asimismo, la mayoría de los hogares utiliza en un $90 \%$ el servicio de recolección de desechos de la zona. Según el autor Ventaja (2006) unas 9000 toneladas diarias de basura asfixian el territorio nacional, distribuidas entre botaderos a cielo abierto $y$ vertederos semicontrolados.

La Universidad de Costa Rica (UCR), se ha comprometido desde sus inicios a formar ciudadanos en la docencia, investigación y acción social, en la búsqueda de opciones que permitan

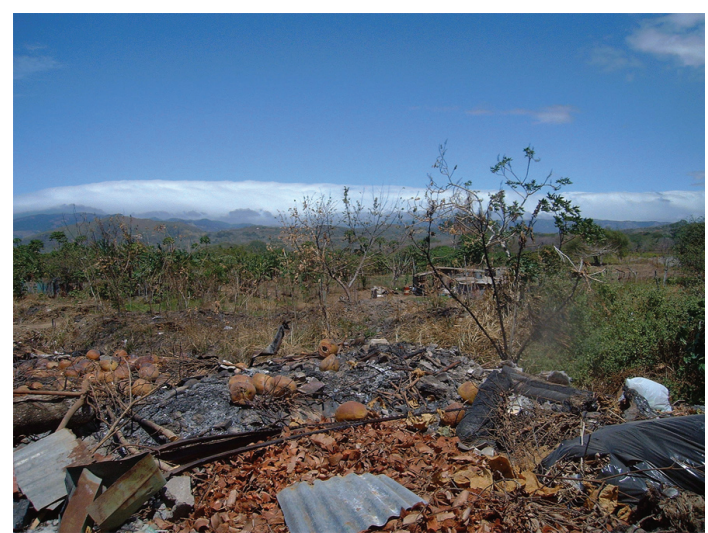

Fotografía 1. Orillas del Río Barranca, nótese la contaminación y quema de árboles por parte, de los residentes de la zona. Febrero 2007. recuperar el desarrollo de la nación. La Acción Social de la UCR pretende, mediante sus trabajos comunales universitarios, transmitir una educación humanística, que permita al estudiante estar en contacto con las comunidades, vivir los problemas sociales $y$ plantear soluciones en una verdadera dimensión de lo humano, por encima del interés práctico.

El Trabajo Comunal Universitario (TCU) que realiza la Sede del Pacífico de la UCR aborda la actividad: Gestión Ambiental al Nivel Comunal, que busca contribuir con la formación de una consciencia crítica ambientalista en la población. Su objetivo principal descansa en destacar la protección del ambiente mediante la sensibilización, la capacitación y la acción con los diferentes actores para mejoramiento del entorno de las comunidades. Este proyecto presenta como objetivos específicos:

1. Promover la participación de la comunidad en el reconocimiento, la promoción, la prevención y atención de problemas ambientales que afectan su entorno.

2. Desarrollar experiencias concretas ambientalistas en las comunidades de Chacarita, Barranca y Puntarenas, junto con actores sociales presentes en ese nivel local, tales como: El Ministerio de Educación, MINAE, InCOPESCA, Ministerio de Salud y Clínicas de Salud, con el fin de propiciar el desarrollo de una conciencia ambientalista en dichas comunidades.

3. Fomentar las iniciativas comunitarias relacionadas con la temática ambientalista y de salud prevaleciente en la zona.

4. Coordinar actividades comunitarias con asociaciones sin fines de lucro, para el fortalecimiento del medio ambiente.

El proyecto se realiza durante 300 horas $y$ es un trabajo multidisciplinario con estudiantes de diferentes carreras: Enseñanza de Inglés, Dirección de Empresas, Informática Empresarial, Enfermería, Turismo Ecológico y otros. Cada grupo TCU recibe una capacitación por 40 horas, en diversos talleres. Incluye información sobre el reglamento y las políticas del TCU.

En esta etapa se les imparte conferencias sobre la contaminación ambiental y la 


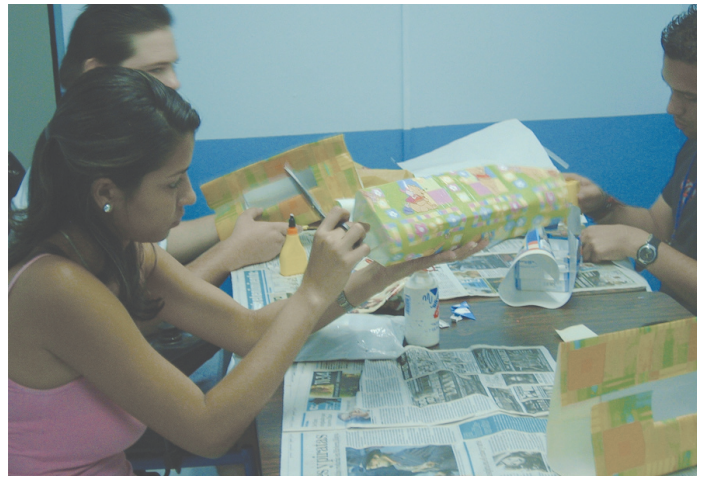

Fotografía 2.Capacitación sobre manualidades con desechos, recibidas por los estudiantes que matriculan el proyecto de TCU, en la Sede del Pacífico. Marzo, 2007

legislación de Costa Rica, con la participación de conferencistas con amplia experiencia en proyectos de ambiente. Asimismo, mediante sesiones $y$ giras didácticas los estudiantes aprenden sobre el manejo adecuado de los desechos sólidos y, por último, reciben talleres para la elaboración de diversas manualidades con desechos que podemos recuperar del ambiente como: cajas de leche, latas de todo tipo, botellas de vidrio, canastas de papel periódico y muchos más. Esta primera etapa es considerada como de formación y capacitación previa, para iniciar el proyecto con miras a lograr la sensibilización e identificación de los estudiantes en las actividades por desarrollar.

El proyecto aborda los siguientes temas:

1. Desarrollo de talleres sobre aprovechamiento de desechos en escuelas, grupos, amas de casa, adultos mayores y otros.

2. Capacitaciones dirigidas a redes comunitarias para la sensibilización del medio ambiente.

3. Limpieza de la playa.

4. Campañas de divulgación contra el dengue y la tuberculosis.

5. Limpieza y ornato de los parques.

6. Colaboración con la existencia de un centro de acopio en la Sede.

7. Colaboración con organizaciones que pretenden mejorar la calidad de vida en materia de ambiente, como la Asociación por Rescate Animal, hogares de ancianos, redes comunitarias, etc.

La interrelación de los alumnos con la comunidad cumple con los objetivos del proyecto, por cuanto los humaniza y los hace más sensibles sobre los problemas sociales que los aqueja, como pobreza, drogadicción, alcoholismo, desempleo, abuso infantil y otros. Además, el desarrollo de talleres de reutilización de desechos en las escuelas y comunidad fomenta un reconocimiento de la sociedad, sobre la importancia del trabajo realizado por los estudiantes de Trabajo Comunal Universitario, lo que promueve una futura fuente de empleo.

En general, esta participación con los niños en las escuelas y las comunidades, $y$ el compartir su entusiasmo en los talleres de manualidades con desechos sólidos o charlas ambientales de la región, permite lograr que sean agentes multiplicadores de la educación ambiental en sus hogares.

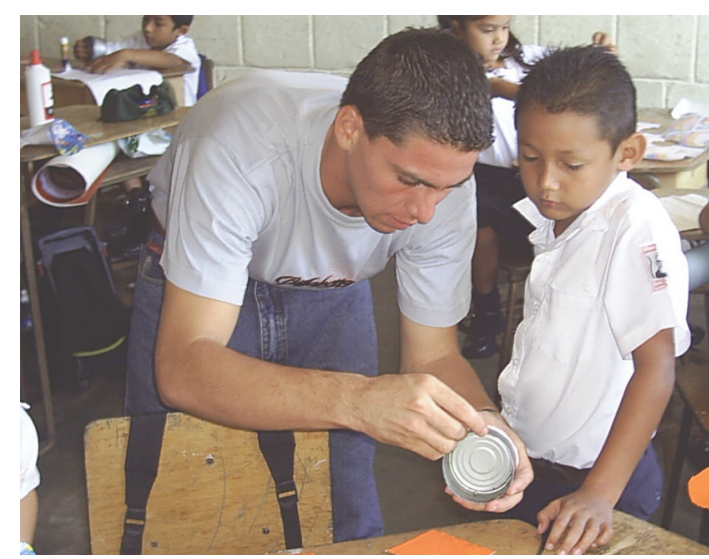

Fotografía 3. Taller en la escuela Fray Casiano de Madrid.

A continuación, se presenta una fotografía de las manualidades realizadas en los talleres educativos en las comunidades:

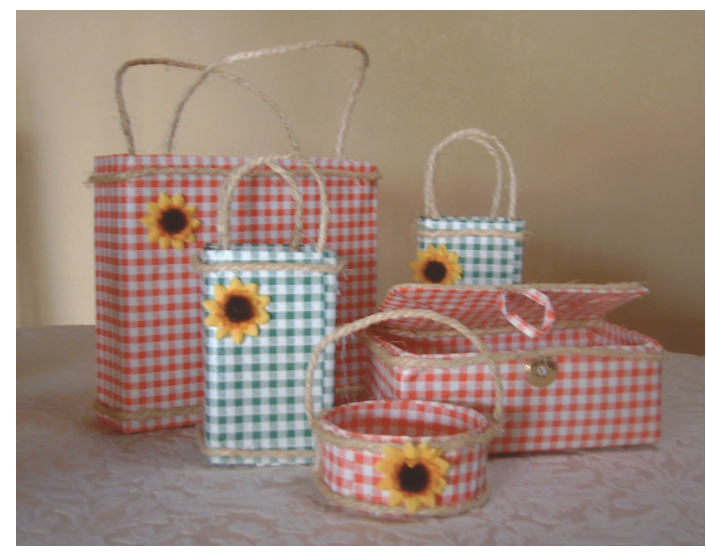

Fotografía 4. Conjunto de Manualidades. 


\section{DIVERSIDAD EN TALLERES Y ACTIVIDADES}

La eficacia del TCU-Gestión ambiental al nivel comunal en el período 2003-2007 se plasma en algunas comunidades con mayores problemas ambientales representadas por: Chacarita, Barranca y Puntarenas (Barrio El Carmen cerca de la Terminal del Ferry).

\section{IMPACTO SOCIAL}

Con el proyecto se ha contribuido a fomentar el interés por el tema del ambiente en la población puntarenense, mediante la coordinación permanente con diferentes actores sociales como el Ministerio de Salud, la Caja Costarricense de Seguro Social, las redes comunitarias, el Instituto Mixto de Ayuda Social, el Ministerio de Educación Pública, la Asociación por Rescate Animal, Acueductos y Alcantarillados, Incopesca y las Asociaciones de Desarrollo de las comunidades.

Seguidamente se mencionan los diversos talleres y las actividades impulsadas y desplegadas por el proyecto para el logro de los objetivos propuestos.

TALLER DE EDUCACIÓN AMBIENTAL EN ESCUELAS PRIMARIAS

En el marco del taller de manualidades con desechos sólidos se coordinó con los directores de centros escolares ubicados en Barranca, Chacarita y Puntarenas, atendiéndose un $98 \%$ de las escuelas. Aproximadamente se han capacitado alrededor de 1000 profesores de enseñanza primaria y en promedio más de 25000 niños durante la vigencia del proyecto. Con el uso del método del aprendizaje práctico (beneficio otorgado) de las $4 \mathrm{R}$ : reducir, rechazar, reutilizar y reciclar (preservar y mejorar el medio ambiente). Los niños se convierten en agentes multiplicadores del mejoramiento del entorno.

En estas labores, la Escuela de Enseñanza Especial del Roble Puntarenas, fue incluida y participó con éxito como una instancia más de acción social que realiza la Universidad de Costa Rica.

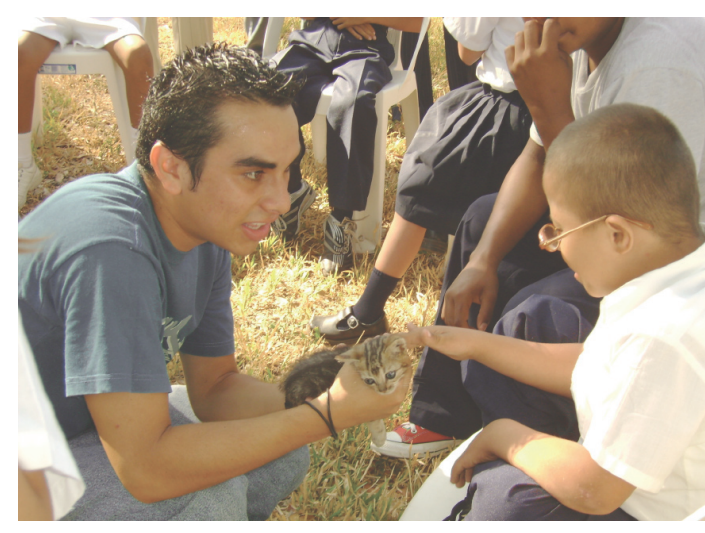

Fotografía 5.Taller educo-ambiental en la Escuela Enseñanza Especial. Febrero, 2007.

$\mathrm{Al}$ respecto se han recogido las opiniones de varios estudiantes que expresan así lo que el programa los ha impactado:

La oportunidad de pasar momentos con los niños de la escuela, me ha hecho valorar aun más la vida; me motiva saber que a pesar de las dificultades de estas personas como la pobreza, se respira un aire de superación y amor por seguir adelante. (Roy Eduardo Guido Lara, A56611, estudiante de Informática Empresarial).

La experiencia vivida en la Escuela de Enseñanza Especial, me ha dejado valorar más todo lo que tengo y he visto que esos niños incluso con sus dificultades, son seres humanos dispuestos a vencer los retos que la vida les depara. (Verónica Ramírez Agüero, A44248, estudiante de Enseñanza del Inglés).

\section{SEGUIMIENTO EN LA COORDINACIÓN DE ACTIVIDADES CON LA RED COMUNITARIA DE CHACARITA, BARRANCA Y PUNTARENAS}

La coordinación de actividades está integrada por personas con interés de trabajar por el bienestar de la comunidad, entre ellos líderes comunales, quienes participan mediante acciones coordinadas con cada Red Comunitaria. Un primer paso es informar a la comunidad de los problemas ambientales. Luego, se puntualiza 
en la capacitación con talleres de información y educación sobre las posibles opciones de tratamiento del entorno.

El beneficio lo han recibido aproximadamente 35000 personas, entre ellas amas de casa, jefes de familias y comunidades. Como resultado, se ha percibido una mayor información y organización de las condiciones ambientales en cada colectividad de Barranca, Puntarenas y Chacarita.

\section{ELABORACIÓN DEL PROYECTO EN MULTIMEDIA PARQUE MARINO (PERÍODO 2005 AL 2006)}

Durante los años 2004 y 2005 se realizó una campaña de promoción en todos los centros escolares y colegiales, sobre la existencia e importancia de la labor de esta institución en la preservación de especies marinas de la zona. Además, en el 2005 y 2006 se ha dado asistencia en mejoramiento de multimedios visuales. Como datos estimados, se han beneficiado con el proyecto cerca de 10000 personas: niños $y$ comunidad en general.

Una de las alumnas opina al respecto:

En este proyecto tuve la oportunidad, de interactuar con personas de diferentes clases sociales, así como mejorar mi perspectiva de los problemas sociales. Lo mejor de todo, es haber adquirido una consciencia crítica que me permitirá ayudar al prójimo, no solo en lo ambiental, sino en una línea más amplia. (Tatiana Benavides Montero, A40754, estudiante de Dirección de Empresas).

ELABORACIÓN DE UNA PÁGINA WEB PARA

LOS HOGARES DE ANCIANOS DE BARRANCA, MIRAMAR Y HERRADURA (2004-2005-2006-2007)

Otra actividad fue la elaboración de la página web para promocionar cada hogar de ancianos, con el objetivo de recaudar fondos venidos del exterior del país. Esto beneficia a más de 3000 personas, entre ellas adultos mayores y comunidad en general.

Este proyecto fue relacionado con visitas a estos centros de adultos mayores, para interesarlos en programas de cultivos de hortalizas y elaboración de talleres de manualidades de reutilización de desechos sólidos.

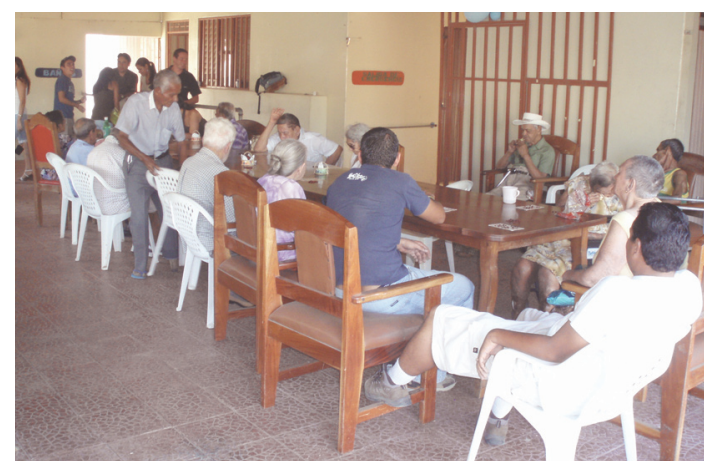

Fotografía 6. Taller educo-ambiental en Hogar de Ancianos de Barranca. Febrero, 2007.

Al respecto, dos estudiantes opinaron:

Esta actividad fue mi favorita, fue una experiencia enriquecedora y maravillosa, ya que me permitió ver cómo hay adultos mayores prácticamente solos, que únicamente tienen a sus compañeros en el Hogar de Ancianos. Pero poseen mucho espíritu para vivir, cosa de la que muchos jóvenes siempre nos estamos quejando. (Yendry Calderón Arrieta, A56602, estudiante de Dirección de Empresas).

Me impactó observar la fortaleza con la que viven estos adultos mayores, a pesar de su soledad $y$ abandono por parte de sus familiares. (Mario Steven Garita Vega, A42234, estudiante de Informática Empresarial).

\section{RECONOCIMIENTO DE LA PROBLEMÁTICA Y LA NECESIDAD DE RECUPERACIÓN DE LA CUENCA DEL RÍO BARRANCA}

Los estudiantes de TCU han realizado extensos recorridos con miembros de la Red Comunitaria en los sitios vulnerables $y$ se les ha planteado la necesidad de recuperar dicha cuenca mediante actividades de mejoramiento ambiental. En cada oportunidad establecen 


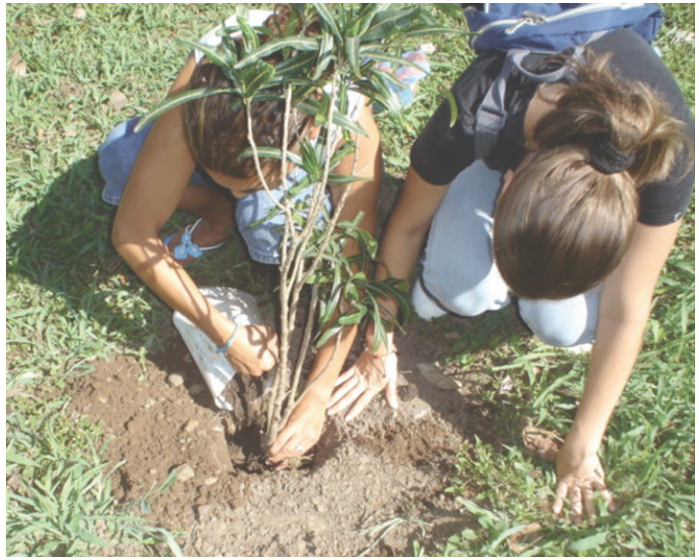

Fotografía 7. Actividad de reforestación.

contactos con Acueductos y Alcantarillados para futuros proyectos de arborización, y han reforestado una parte de una orilla del río con el consecuente beneficio para más de 15000 personas.

Esta joven expresó su parecer:

El TCU nos hace conocer nuestro lado más humano, al ponernos de frente ante las situaciones extremas de la realidad nacional. Nos brinda la oportunidad de reflexionar y concienciar acerca de la problemática ambiental que actualmente se está viviendo en la provincia de Puntarenas. (Diana Marenco Guevara, A33002, estudiante de Dirección de Empresas).

\section{PARTICIPACIÓN EN LA LIMPIEZA DE PLAYAS}

Estas actividades se han coordinado con INCOPESCA y el Parque Marino del Pacífico y, en otras, con las Municipalidades de Puntarenas y Esparza.

Así opinó un joven universitario:

Al interactuar con personas de distintas clases sociales, pude llegar a una conclusión: es mentira que la culpa de la contaminación sea de los pobres, ellos son unas víctimas más de los problemas sociales. Durante el proyecto, quienes eran más humildes colaboraban más en soluciones ambientales. (Olman Carvajal
Atencio, A21158, estudiante de Dirección de Empresas).

JORNADA DE ARBORIZACIÓN EN EL ÁREA DE LA
PLANTA DE TRATAMIENTO AGUAS NEGRAS
DE ACUEDUCTOS Y ALCANTARILLADOS

Esta actividad contó con una eficaz coordinación de acciones y logró la siembra de un total de 100 árboles, principalmente aromáticos. Los estudiantes también participaron en el cuido y mantenimiento de los que estaban sembrados.

Un alumno de Enfermería puntualizó:

Este TCU me permitió conocer las zonas más marginales del cantón de Puntarenas, así como la brecha social entre las poblaciones más sobresalientes. Logró inclusive sobrepasar mis expectativas acerca de un Trabajo Comunal, y llegué a concluir que entre todos podemos mejorar la calidad de vida que llevamos. (Julio César Montero Rojas, A06234, estudiante de Enfermería).

\section{JORNADA DE CAMPAÑAS DE PROMOCIÓN Y DIVULGACIÓN DE LA ASOCIACIÓN POR RESCATE ANIMAL}

Las campañas de promoción y divulgación realizadas por la Asociación por Rescate Animal Puntarenas en los distritos de Barranca, Puntarenas y Chacarita, lograron controlar la sobrepoblación de animales caninos y felinos en las zonas de influencia de la Sede. Asimismo, mejoraron las condiciones sanitarias y ambientales de perros y gatos de los distritos abarcados por el proyecto, en relación con los animales que deambulan sin dueño por las calles, donde abren las bolsas de basura y comen alimentos, muchas veces en descomposición. Ahora bien, según la doctora Soto (presidenta de la Asociación), con los censos realizados por los estudiantes de TCU, se ha obtenido, por primera vez en Costa Rica, datos sobre las condiciones y la cantidad de animales en esta situación en las comunidades de Chacarita, Barranca $y$ Puntarenas. 


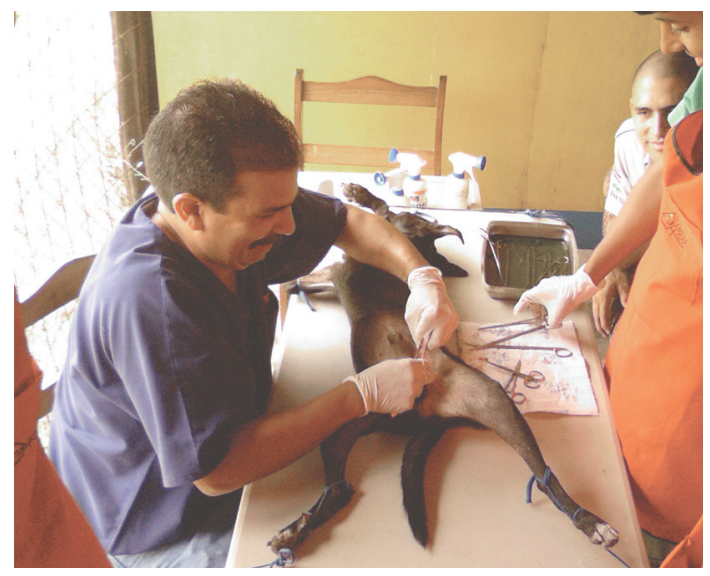

Fotografía 8. Cirugía de castración realizada por el veterinario Francisco Gómez y asistida por un estudiante de TCU. Noviembre, 2006.

Estudiantes que han finalizado el TCU son miembros de la Asociación por Rescate Animal y hoy cumplen una labor significativa como miembros activos en la sociedad puntarenense.

Se calcula que existen 40000 personas beneficiadas con estas acciones, entre ellas: amas de casa, jefes de hogar, niños, etc. de las localidades de Chacarita, Barranca, Puntarenas, Miramar, Esparza y La Isla de Chira. Esos habitantes en la actualidad, "reconocen la castración como el método de control de población de perros y gatos no deseados".

Un alumno expresó esta experiencia:

El TCU me permitió sentirme cómodo realizando los talleres en las escuelas $y$ los Jardines de Niños en los que participé. Debemos seguir impulsando en la niñez esta cultura de reutilizar los desechos sólidos, para que así ellos puedan sensibilizar a sus padres y demás familiares en cuanto a la importancia de la conservación de nuestro medio ambiente.

Por otro lado, logré comprender el esfuerzo que realizan las personas de la Asociación por Rescate Animal (APRAP), para reducir la sobrepoblación de los animales que son víctimas del abandono. Es lastimoso observar el sufrimiento de animales como perros y gatos que deambulan por las calles en busca de alimento abriendo bolsas de basura y contaminando. (Jonathan Delgado Cruz, A56609, estudiante de Informática Empresarial).

PARTICIPACIONES EN FERIAS DE LA SALUD,

FERIAS DEL AGRICULTOR Y EXPO

EN LA SEDE RODRIGO FACIO

Todos los años el grupo de TCU participa en las ferias de la Salud, organizadas por la Caja Costarricense de Seguro Social para los diferentes distritos. Este equipo da a conocer los problemas del medio ambiente $y$, también ayuda en la búsqueda de soluciones. Además, en la Feria del Agricultor de Barranca y en la EXPO en la Sede Rodrigo Facio, se han promocionado las labores de los estudiantes de TCU efectuadas mediante diversas actividades, con resultados muy halagadores para continuar con el proyecto. Aproximadamente, se presume que cerca de 50000 personas tales como niños, mujeres, hombres $y$ adultos mayores han recibido estos conocimientos.

Al respecto, un alumno de Enfermería aseguró que:

Me siento satisfecho de haber escogido este TCU, como estudiante de enfermería en la Sede Rodrigo Facio, hubiese podido escoger cualquier otro proyecto de San José. Pero, gracias a Dios, acerté y matriculé uno de los más enriquecedores a nivel social y ambiental que tiene la Universidad de Costa Rica. (Augusto César Vado Acuña, A25182, estudiante de Enfermería).

\section{JORNADAS DE CAMPAÑAS DE PROMOCIÓN Y DIVULGACIÓN CONTRA EL DENGUE}

Este TCu colabora junto con el Ministerio de Salud, la Caja Costarricense de Seguro Social y la Asociación por Rescate Animal en los distritos de Barranca, Chacarita y Puntarenas en dos actividades: 1) En la lucha contra los criaderos del mosquito del dengue y la malaria, 2) en la limpieza sanitaria. Las poblaciones beneficiadas 
suman alrededor de 30000 personas (niños, mujeres, hombres $y$ adultos mayores).

En esta participación, una joven opinó:

El TCU me permitió enseñarles a las personas, tanto a niños como adultos, la importancia de implementar en nuestras vidas una mejor manera de aprovechar los desechos sólidos $y$ hacerles ver lo importante de castrar tanto perros como gatos. También recordarles a las personas el aseo que deben tener en diversas épocas del año donde nos acechan enfermedades producidas por mosquitos como es el caso del dengue. Si cada uno revisa sus alrededores, nos podríamos evitar la muerte de un vecino o un ser querido. (Mayra Benavides Villalobos, A30742, estudiante de Dirección de Empresas).

\section{JORNADAS DE CLASIFICACIÓN} DE DESECHOS SÓLIDOS

Mediante campañas de divulgación y promoción, los estudiantes han educado a las comunidades beneficiadas del proyecto, para la clasificación y recolección de los desechos sólidos en conjunto con la Asociación de Coopeprogreso (grupo de mujeres jefas de hogar, que realizan limpiezas de playa $y$ recolectan desechos sólidos como fuente de empleo), la cual está ubicada en las instalaciones de la Sede. En forma estimada, se supone que las actividades han favorecido a más de 25000 personas de la sociedad de Puntarenas (Barranca, Chacarita y Puntarenas).

Un alumno de Informática expresó al respecto:

De alguna manera espero que mi pequeña contribución haya servido de algo para hacer que esta provincia mejore en materia de ambiente. La educación ambiental tiene que ir de la mano, por el bien de todos los que habitamos en esta hermosa tierra. (Leroy Gómez Romero, A56610, estudiante de Informática Empresarial).

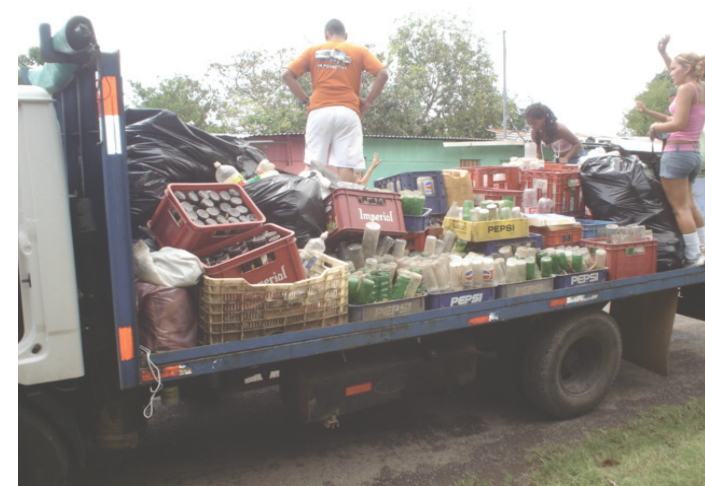

Fotografía 9: Campaña de recolección de desechos sólidos en Barranca, Puntarenas y Chacarita por estudiantes de TCU.

Y Luisa Hernández advirtió:

En el TCU, de todos los proyectos realizados con los que más me identifiqué fueron con los del reciclaje de desechos sólidos y con los hogares comunitarios. El primero, porque se trata de proteger el medio ambiente de tanta contaminación que todos producimos y considero relevante una reutilización de estos productos. Segundo, hay que reconocer el apoyo que la Universidad brinda a las señoras del Centro de Acopio al darles un espacio ahí, para que ellas puedan hacer su trabajo, ya que son jefas de hogar y el reciclar contribuye a su fuente de ingreso. Gracias a la Sede del Pacífico, a la profesora coordinadora y a la comunidad por brindarnos esta experiencia. (Luisa Hernández Lo, 84J195, estudiante de Enseñanza en el Inglés).

\section{ACTIVIDADES CON LA MUNICIPALIDAD DE PUNTARENAS}

Mediante el aseo y ornato de parques, se ha logrado crear conciencia en la limpieza y rotulación de estos lugares. En este momento, se hace muy poco esta labor dentro del proyecto de TCU, porque las comunidades y la Municipalidad se han responsabilizado de estos bienes públicos y ellos les dan el mantenimiento necesario. En su oportunidad, se benefició a más de 30000 personas. 
Al respecto, un joven estudiante de Informática puntualiza:

Aprendí muchas cosas, en primera instancia a no juzgar a un libro por su portada, mi visión de Chacarita era la de un pueblo ruin y peligroso. Pero, di un giro de $90^{\circ}$ al conocer $y$ charlar con sus habitantes, me contaron historias $y$ fueron mis amigos. Me fui dando cuenta de que es un pueblo como cualquier otro. (Luis Fernández Soto, A31985, estudiante de Informática Empresarial).

Y una estudiante comentó lo siguiente:

Este proyecto de TCU permite que el profesional se prepare no solo académicamente, sino que enriquezca su persona con valores de cooperación, solidaridad, respeto, lealtad, honradez y tener un panorama más amplio sobre posibles soluciones que debemos emprender en la parte ambiental, como ciudadanos de Costa Rica. Gracias. (Aracelly Palma Moreno, A33869, estudiante de Dirección de Empresas).

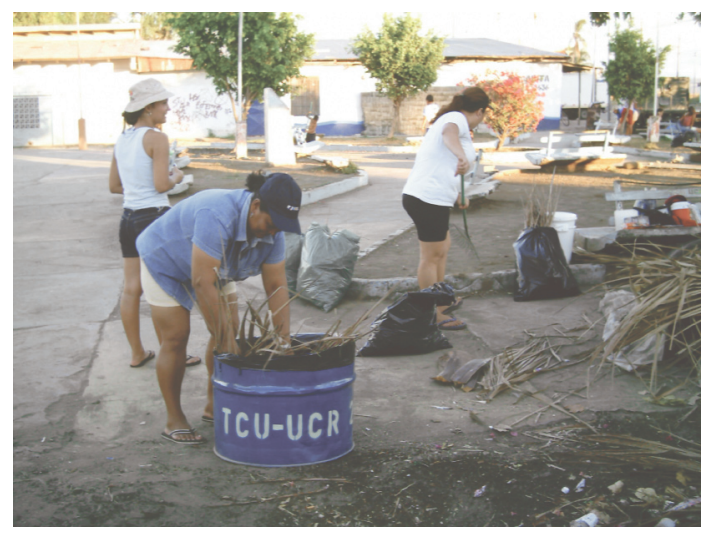

Fotografía 10. Limpieza y ornato de parques de Chacarita.

\section{RESULTADOS}

El desarrollo de las acciones anteriormente descritas, presenta resultados reveladores:

$\diamond$ Una mayor presencia institucional de forma sostenida en las comunidades de trabajo, para lo cual en horas estudiante se ha invertido desde el año 2003 al 2006 un total de 85800 horas comunitarias.

CUADRO 1

LISTA DE ESTUDIANTES QUE APRUEBAN EL TCU AÑOS 2003 Y 2006

\begin{tabular}{lcc}
\hline AÑO & $\begin{array}{c}\text { Nro. de estudiantes que } \\
\text { aprobaron TCU }\end{array}$ & $\begin{array}{c}\text { Horas de TCU en } \\
\text { comunidades }\end{array}$ \\
\hline 2003 & 55 & 16500 \\
2004 & 81 & 24300 \\
2005 & 90 & 27000 \\
2006 & $60^{4}$ & 18000 \\
Total & 286 & 85800 \\
\hline
\end{tabular}

El proyecto cuenta con la participación de estudiantes de las carreras antes citadas y en algunas ocasiones hemos contado con disciplinas como Enseñanza en Orientación, Enfermería, Trabajo Social, etc.

$\diamond$ Existe un mayor vínculo con otras instituciones públicas y privadas de la zona, dada la coordinación establecida en el desarrollo de acciones en favor de la salud pública; asimismo se ha procurado la coordinación de esfuerzos comunales.

$\diamond$ En el monitoreo de las acciones del proyecto se ha logrado estructurar un registro de actores sociales con su correspondiente caracterización, que ha permitido una coordinación estrecha de acciones.

$\diamond$ La supervisión de campo por parte de la coordinación del TCu favorece la validación de las actividades.

\section{CAMBIOS IMPORTANTES}

El proyecto permite a los estudiantes de Trabajo Comunal Universitario la oportunidad

4 La diferencia de los años 2005 y 2006 (90$60=30$ ) corresponde a un grupo de estudiantes de Educación Primaria, originada por un Convenio Colegio Universitario de Puntarenas que ya terminó. 
de relacionarse con la comunidad puntarenense y comprender la problemática social que vive la región.

El proyecto Gestión Ambiental al nivel comunal ratifica las siguientes situaciones:

\& Logra una experiencia positiva para los alumnos de TCU, en cuanto a que los posibilita a lograr un equilibrio psicológico, maduración afectiva y socialización con el mundo que los rodea.

$\diamond$ Permite el trabajo en equipo en forma efectiva, mediante las labores realizadas a favor del medio ambiente (no existen actividades individuales).

$\diamond$ Despierta talentos creativos en estudiantes mediante las manualidades de los desechos sólidos en los talleres educoambientales.

Los talleres son una forma de incentivar al niño, a no contaminar, reducir los desechos del ambiente $y$ divertirse. En un taller realizado en agosto del 2006, en una escuela, un niño dijo lo siguiente:

Randy: —Niña, ¿¿de qué trabaja usted en la Universidad? Usted fue mi maestra el año pasado, ¿no se acuerda?

Respuesta: - ¡Así!.... ¿quué clase le di?

Randy: - Usted me enseñó a forrar latas. Ahora trato de forrarlas. ¿Cuándo vuelve a venir?

Respuesta: - Muy pronto, le daré otra vez clases...

En ese sentido, el proyecto deja una huella memorable en los niños que participan en las actividades de Trabajo Comunal Universitario, es una de las tantas experiencias que no se olvidan. Otro niño Reiner, de aproximadamente 6 años (el cual tenía cáncer y estaba en tratamiento de quimioterapia, incluso no tenía cabello, situación que disimulaba con una gorra) se acercó hacia mi persona en un taller, $y$ dijo:

Reiner: - Maestra, ¿Puedo hacer el taller con usted?
Respuesta: - Sí, claro, acomódese aquí. Reiner: - Vengo a trabajar bien, porque todo lo que hago debe estar bien, para eso me mandó Dios.

Cuando terminó expresé:

— ¡La latita y la caja de leche forrada, le quedaron muy lindas!

Reiner: -Vuelva, pronto maestra, me divertí mucho ( $y$ me dio un abrazo).

Respuesta: - Muy pronto lo volveré a ver...

La realización del Trabajo Comunal Universitario permite vivir muchas situaciones diversas, como las que se detallan:

$\diamond$ Muchos niños de comunidades pobres llegan al centro educativo con sus necesidades de alimentación insatisfechas. En los años 2004 y 2005 algunos educandos de la enseñanza primaria del Barrio de Chacarita sufrían desmayos durante los talleres del Tcu. Por tal razón, las autoridades universitarias de Acción Social, a partir del 2006, autorizan un pequeño refrigerio y ofrecen el $90 \%$ de los materiales utilizados por el TCU en las escuelas públicas de bajos recursos de esta comunidad.

$\diamond$ En ocasiones las personas confunden el TCU como una fuente de mano de obra gratis, por ejemplo, solicitan verbalmente arreglar un caño o limpiar un lote baldío.

$\diamond$ El embellecimiento de los parques se realiza con el propósito de contribuir con el aseo, ornato y conservación del medio que nos rodea. Recientemente las comunidades de los Barrios El Carmen, Chacarita y Barranca han tomado conciencia y se han organizado para cuidar estos bienes públicos.

$\diamond$ Aún existen en las comunidades de Chacarita, Barranca y Puntarenas, personas no solo con poca conciencia de los daños ocasionados a la naturaleza, sino también sin disposición para repararla a través del tratamiento de los desechos sólidos.

Según las encuestas realizadas a la población beneficiada del proyecto a principios del 2007, este Trabajo Comunal Universitario, avanza por las siguientes razones: 
Impulsa el aseo, reforestación y ornato de parques públicos, e interesa a las comunidades de hacerse responsables del mantenimiento de estos.

Fomenta la protección del medio ambiente. El TCU transmite aprendizajes importantes en materia de ambiente con lo cual logra sensibilizar a la sociedad puntarenense beneficiada.

Posibilita la reutilización de los desechos sólidos. Permite al individuo implementar nuevos usos a estos materiales. Estos podrían representar una fuente de ingreso para la familia en un futuro.

Educa a la comunidad en general sobre el mejoramiento ambiental.

La Universidad de Costa Rica se proyecta a la comunidad en una forma positiva. La labor del TCU no pasa inadvertida por los residentes de Puntarenas, quienes muestran interés en estas actividades. Por ejemplo, para reciclar hacen llegar botellas de vidrio, papel periódico, botellas de plástico, etc. al centro de Acopio administrado por Coopeprogreso en las instalaciones de la Sede del Pacífico.

Integra a toda una comunidad en el problema ambiental y sus posibles soluciones.

Son muy gratificantes las observaciones

que establecen los beneficiarios del proyecto, como las siguientes:

Felicitan e instan a continuar siempre con este tipo de proyecto como talleres en escuelas, campañas de castración de animales, talleres para adultos mayores, reforestaciones, etc.

Proponen la inclusión de las escuelas en los beneficios de los talleres de reutilización de desechos. Se aclara que para estas actividades la Universidad como tal, dentro del presupuesto de TCU, brinda todos los materiales a las escuelas de bajos recursos como: mecates, goma, papel de regalo, silicona y otros, los cuales son usados racionalmente.

Consideran que el aporte de los estudiantes es valioso porque permite una información precisa para diversas entidades: el Ministerio de Salud, la Clínica de Salud, Incopesca, Asociación por Rescate Animal y otros. Así por ejemplo, en el caso del Ministerio de Salud, además de la campaña de prevención contra el dengue, censan a los habitantes que no deseaban recibir información.

Refuerzan el avance en la proyección de los valores ambientales. Hay que seguir con el énfasis en el cuido del medio ambiente, porque todavía existen personas que lo contaminan.

\section{REFLEXIONES FINALES}

La protección al ambiente más que un discurso debe convertirse en una práctica para la sociedad, vista como una forma de prolongar nuestra vida. Los valores ecológicos no bastan con enseñarse, sino que es necesario llevar a la práctica entornos limpios y protegidos con personas que desean salvaguardar y respetar el ambiente. Por lo anterior, urge una ley sobre la clasificación de los desechos sólidos en nuestros hogares, para tomar medidas de protección al medio ambiente. Las empresas deben comprender que al escasear las materias primas y suministros de energía, se hacen imprescindibles los materiales de segunda mano y su respectivo reciclaje.

Muchas naciones industrializadas como Alemania, Inglaterra, Holanda, Suiza, Estados Unidos, etc., hablan sobre su reconstrucción ecológica, pero para los países pobres cuando se pierde su riqueza natural o se altera irreversiblemente el equilibrio de la naturaleza, es difícil predicar la reconstrucción. Entonces, encontrar desde ahora una solución adecuada para la conservación es un desafío de todo costarricense.

Porque la calidad de vida en el ámbito nacional tiene que convertirse en un factor 
importante de planificación gubernamental, $y$ no se debe esperar un revés económico o catástrofe para que los gobernantes actúen; es prioritaria una educación ambiental como política obligatoria impostergable para todas las generaciones.

Por lo anterior, es angustioso que las comunidades de Barranca, Puntarenas y Chacarita, beneficiadas con este proyecto TCU 461-UCR, presenten de una u otra forma algún problema de ambiente; por la falta de conciencia sobre la protección de los recursos naturales. Así por ejemplo, el río Barranca sigue presentando una disminución de su caudal debido a la deforestación y contaminación, luego Chacarita

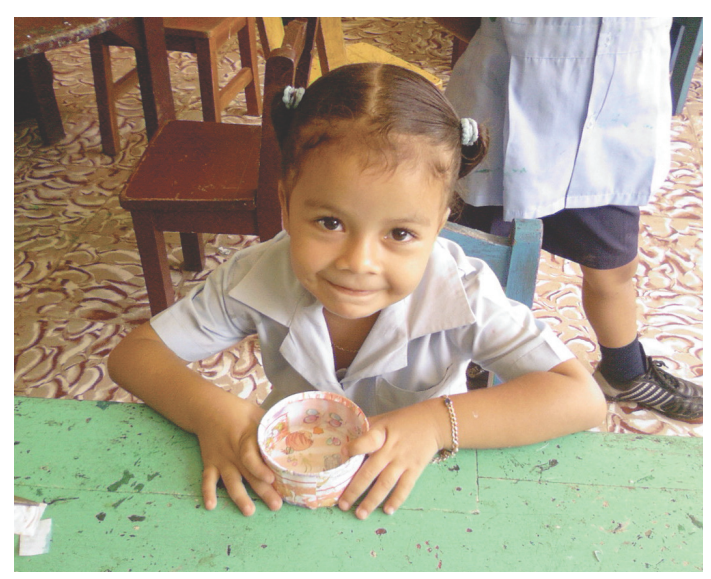

Fotografía 11.Taller educo-ambiental en el Kinder de Fray Casiano de Madrid en Chacarita.

continúa con serios problemas de basura $y$ suciedad; por último, Puntarenas enfrenta problemas de contaminación ambiental en la playa.

En realidad, todavía existe mucho por hacer en relación con la preservación del medio ambiente; esta gestión ambiental debe seguir colaborando para que futuras generaciones sean conscientes de que en algunos casos el llamado progreso urbano daña a la madre naturaleza.

La Sede del Pacífico debe seguir desarrollando este TCU, porque si bien es cierto que en ocasiones existe irreflexión ambiental por parte de la población, los talleres de reutilización de desechos en las escuelas dan una esperanza de poder moldear a los adultos del mañana, para que continúen con las prácticas de proteger los recursos naturales. Así también la disminución de la población canina no deseada permite disminuir el maltrato hacia esta clase de animal y la reducción de la contaminación casera.

Existe un gran reto para el milenio, al procurar soluciones sanas y amigables para el ambiente. No obstante, queda en manos del Gobierno, políticos, organismos, mujeres $y$ hombres de buena voluntad optar por una calidad de vida mejor.

\section{REFERENCIA BIBLIOGRÁFICA}

Diccionario de la Real Academia de la Lengua Española. $22^{\circ}$ Edición. 2001. En: <www. rae.es>

OMS/PNUMA. "Las ciudades más contaminadas del mundo". En: <http: //www.ecoestrategia.com/artículos/ mapas/artículos/mapas02.html>

Arce, S. "El aporte de Costa Rica". Periódico La Nación. San José, Costa Rica. 13 de febrero del 2005: $16 \mathrm{~A}$.

Mediavilla, P. "Salvar las cuentas". Periódico La Nación, Proa. San José, Costa Rica. 04 de febrero del 2007: 11.

Sierra, K. "Agua para todos". Periódico La Nación. San José, Costa Rica. 12 de marzo del 2006: $34 \mathrm{~A}$.

Ventaja, C. "9 000 toneladas de basura asfixian al país". Periódico La Nación. San José, Costa Rica. 02 de abril del 2006: $4^{\mathrm{a}}$. 
